\title{
Isoperimetry for spherically symmetric log-concave probability measures
}

Nolwen Huet

\begin{abstract}
We prove an isoperimetric inequality for probability measures $\mu$ on $\mathbb{R}^{n}$ with density proportional to $\exp (-\phi(\lambda|x|))$, where $|x|$ is the euclidean norm on $\mathbb{R}^{n}$ and $\phi$ is a non-decreasing convex function. It applies in particular when $\phi(x)=x^{\alpha}$ with $\alpha \geq 1$. Under mild assumptions on $\phi$, the inequality is dimension-free if $\lambda$ is chosen such that the covariance of $\mu$ is the identity.
\end{abstract}

\section{Introduction}

In his paper [10], Bobkov studies the spectral gap for spherically symmetric probability measures $\mu$ on $\mathbb{R}^{n}$ with density

$$
\frac{d \mu(x)}{d x}=\rho(|x|),
$$

where $\rho$ is log-concave. His main result can be stated as follows.

Theorem 1 (Bobkov [10]). The best constant $P_{\mu}$ in the Poincaré inequality

$$
\operatorname{Var}_{\mu}(f) \leq P_{\mu} \int|\nabla f|^{2} d \mu, \quad \forall f \text { smooth }
$$

satisfies

$$
\frac{\mathrm{E}_{\mu}\left(|X|^{2}\right)}{n} \leq P_{\mu} \leq 12 \frac{\mathrm{E}_{\mu}\left(|X|^{2}\right)}{n} .
$$

In particular, if $\mu$ is isotropic, we get

$$
1 \leq P_{\mu} \leq 12,
$$

which means a spectral gap not depending on $n$.

2000 Mathematics Subject Classification: 26D10, 60E15, 28A75.

Keywords: Isoperimetric inequalities, log-concave measures. 
Remark. Actually the constant he obtained was 13, but bounding two positive constants by their maximum instead of their sum in his proof, leads to the slightly better constant 12 .

Here " $\mu$ is isotropic" means that the covariance of $\mu$ is the identity. However, we already know from the spherically invariance of $\mu$ that the covariance is proportional to the identity. So in our case, the isotropy of $\mu$ reduces merely to $\mathrm{E}_{\mu}\left(|X|^{2}\right)=n$.

If we assume furthermore that $\mu$ itself is log-concave (see [11] for precisions about $\log$-concave measures), that is to say that $\rho$ is non-decreasing, then one can deduce an isoperimetric inequality for $\mu$, thanks to a result of Ledoux [18] (generalized in [20] by E. Milman) bounding the Cheeger constant from below by the spectral gap.

Theorem 2. There exists a universal constant $c>0$ such that, for any $n \in \mathbb{N}$, all $\log$-concave measures $\mu$ on $\mathbb{R}^{n}$ spherically symmetric and isotropic satisfy the following isoperimetric inequality:

$$
\operatorname{Is}_{\mu}(a) \geq c a \wedge(1-a) .
$$

Here $\operatorname{Is}_{\mu}$ denote the isoperimetric function of $\mu$ and $a \wedge b=\min (a, b)$. We need some notation to define $\mathrm{Is}_{\mu}$ properly. Let $A$ be a Borel set in $\mathbb{R}^{n}$. We define its $\varepsilon$-neighborhood by

$$
A_{\varepsilon}=\{x \in X ; d(x, A) \leq \varepsilon\} .
$$

The boundary measure of $A$ is

$$
\mu^{+}(\partial A)=\liminf _{\varepsilon \rightarrow 0^{+}} \frac{\mu\left(A_{\varepsilon}\right)-\mu(A)}{\varepsilon} .
$$

Now the isoperimetric function of $\mu$ is the largest function $\operatorname{Is}_{\mu}$ on $[0,1]$ such that for all Borel sets $A$,

$$
\mu^{+}(\partial A) \geq \operatorname{Is}_{\mu}(\mu(A))
$$

The result of Bobkov answer the conjecture of Kannan, Lovász and Simonovits [14] in the particular case of spherically symmetric measures. This conjecture asserts that (1.1) is true for all log-concave and isotropic measures $\mu$, with a universal constant $c$.

Our aim is to sharpen Theorem 2 when $\rho$ is "better" than log-concave. For instance, the Gaussian measure $\gamma_{n}$ corresponding to $\rho(t)=(2 \pi)^{-\frac{n}{2}} \exp -\frac{t^{2}}{2}$, is known to satisfy the log-Sobolev inequality and the following isoperimetric inequality:

$$
\operatorname{Is}_{\gamma_{n}}(a) \geq c(a \wedge(1-a)) \sqrt{\log \frac{1}{a \wedge(1-a)}}
$$


with constants not depending on $n$ either. We can ask what happens for regimes between exponential and Gaussian, or beyond the Gaussian case. This idea has already been developed in $[16,2,7,5,6]$ for product measures.

Let $\phi: \mathbb{R}^{+} \rightarrow \mathbb{R}^{+}$be a convex non-decreasing function of class $\mathcal{C}^{2}$ such that $\phi(0)=0$. Then we consider the probability measure on $\mathbb{R}^{n}$

$$
\mu_{n, \phi}(d x)=\frac{e^{-\phi(|x|)} d x}{Z_{n, \phi}}
$$

and its associated radial measure on $[0,+\infty)$

$$
\nu_{n, \phi}(d r)=\left|\mathbb{S}^{n-1}\right| \frac{r^{n-1} e^{-\phi(r)} d r}{Z_{n, \phi}}
$$

We denote by $\sigma_{n-1}$ the uniform probability measure on the unit sphere $\mathbb{S}^{n-1}$ of $\mathbb{R}^{n}$. If $X$ is a random variable of law $\mu_{n, \phi}$, then $|X|$ has the distribution $\nu_{n, \phi}$. Conversely, if $r$ and $\theta$ are independent random variables whose distributions are respectively $\nu_{n, \phi}$ and $\sigma_{n-1}$, then $X=r \theta$ has the distribution $\mu_{n, \phi}$. In view of this representation, we will derive inequalities for $\mu_{n, \phi}$ from inequalities for $\nu_{n, \phi}$ and $\sigma_{n-1}$.

In the particular case $\phi(x)=\phi_{\alpha}(x)=x^{\alpha}$ with $\alpha \geq 1$, we note $\mu_{n, \alpha}=$ $\mu_{n, \phi_{\alpha}}$. Between the exponential and the Gaussian case, following the results for $\mu_{1, \alpha}^{\otimes n}$ and $\mu_{1, \phi}^{\otimes n}$ from [6], we expect to bound from below the isoperimetric function of $\mu_{n, \alpha}$ on $[0,1 / 2]$ by

$$
L_{\alpha}(a)=a\left(\log \frac{1}{a}\right)^{1-\frac{1}{\alpha}}
$$

and more generally the one of $\mu_{n, \phi}$ by

$$
L_{\phi}(a)=\frac{a \log \frac{1}{a}}{\phi^{-1}\left(\log \frac{1}{a}\right)} .
$$

On the other hand, since we are aiming at results which do not depend on $n$ and because of the Central-Limit Theorem [15], we cannot expect better isoperimetric profile than the one of the Gaussian measure, proportional to

$$
L_{2}(a)=a \sqrt{\log \frac{1}{a}}
$$

The point is to know the exact dependence in $n$ of the constant in front of the term in $a$, and in particular to know whether we recover universal constants in the isotropic case. The main results of this paper are stated next. The first theorem concerns the special case $\phi(x)=(\lambda x)^{\alpha}$. For every $\alpha \geq 1$ 
and $n \in \mathbb{N}^{*}$, we define $J_{n, \alpha}$ as a continuous function on $[0,1]$, symmetric around $1 / 2$, by

$$
J_{n, \alpha}(a)= \begin{cases}n^{1 / \alpha-1 / 2} a\left(\log \frac{1}{a}\right)^{1-1 / \alpha} & \text { if } 0 \leq a \leq e^{-n} \wedge \frac{1}{2}, \\ a \sqrt{\log \frac{1}{a}} & \text { if } e^{-n} \wedge \frac{1}{2} \leq a \leq 1 / 2 .\end{cases}
$$

Theorem 3. Let $\alpha \geq 1$ and $n \in \mathbb{N}^{*}$. Let $\mu$ be the isotropic probability measure on $\mathbb{R}^{n}$ of density proportional to $e^{-|\lambda x|^{\alpha}}$, with $\lambda>0$. Then, there exists a universal constant $C>0$ such that

$$
\forall a \in[0,1], \quad \operatorname{Is}_{\mu}(a) \geq C J_{n, \alpha}(a) .
$$

In particular,

$$
\forall a \in[0,1], \quad \operatorname{Is}_{\mu}(a) \geq C(a \wedge(1-a))\left(\log \frac{1}{a \wedge(1-a)}\right)^{1-\frac{1}{\alpha \wedge 2}} .
$$

It can be seen as a corollary of Theorem 4 on more general functions $\phi$. Let us introduce the different assumptions made on $\phi$ before stating it.

Hypothesis (H0) $\phi: \mathbb{R}^{+} \rightarrow \mathbb{R}^{+}$is a non-decreasing convex function of class $\mathcal{C}^{2}$ such that $\phi(0)=0$.

Hypothesis (H1) $\phi$ satisfies (H0) and $x \mapsto \phi(x) / x^{2}$ is non-increasing.

Hypothesis (H2) $\phi$ satisfies (H0) and $x \mapsto \phi(x) / x^{2}$ is non-decreasing.

Hypothesis $(\mathbf{H} 2(\boldsymbol{\alpha}, \boldsymbol{\beta})) \phi$ satisfies $(\mathrm{H} 0)$ and there exists $\alpha \geq \beta \geq 2$ such that $x \mapsto \phi(x) / x^{\alpha}$ is non-increasing and $x \mapsto \phi(x) / x^{\beta}$ is non-decreasing.

Let $n \in \mathbb{N}^{*}, \phi$ be a function satisfying (H0), and $J_{n, \phi}$ be a continuous function on $[0,1]$, symmetric around $1 / 2$, and defined on $[0,1 / 2]$ by

$$
J_{n, \phi}(a)= \begin{cases}\frac{\phi^{-1}(n)}{\sqrt{n}} \frac{a \log \frac{1}{a}}{\phi^{-1}\left(\log \frac{1}{a}\right)} & \text { if } a \leq e^{-n}, \\ a \sqrt{\log \frac{1}{a}} & \text { if } a \geq e^{-n} .\end{cases}
$$

For every $\lambda>0$, let $\phi_{\lambda}$ be the function defined by $\phi_{\lambda}(x)=\phi(\lambda x)$. Remark that there exists always $\lambda$ such that $\mu_{n, \phi_{\lambda}}$ is isotropic.

Theorem 4. - Let $\phi$ be a function satisfying $(\mathrm{H} 1)$ or $(\mathrm{H} 2)$, and $n \in \mathbb{N}^{*}$.

Then, there exists a universal constant $C>0$ such that

$$
\forall a \in[0,1], \quad \operatorname{Is}_{\mu_{n, \phi}}(a) \geq C \frac{\sqrt{n}}{\phi^{-1}(n)} J_{n, \phi}(a) .
$$


- Let $\phi$ be a function satisfying (H1), $\alpha=2$, and $\beta=1$, or $\phi$ be a function satisfying $(\mathrm{H} 2(\alpha, \beta))$ with $\alpha \geq \beta \geq 2$. If we choose $\lambda>0$ such that $\mu_{n, \phi_{\lambda}}$ is isotropic, then there exists universal $n_{0} \in \mathbb{N}^{*}$ and $C>0$ such that, for every $n \geq n_{0}$,

$$
\forall a \in[0,1], \quad \operatorname{Is}_{\mu_{n, \phi_{\lambda}}}(a) \geq C(1 / \alpha)^{1 / \beta} J_{n, \phi}(a) .
$$

We can also deduce isoperimetric inequalities not depending on the dimension $n$ anymore.

Theorem 5. Let $n \in \mathbb{N}^{*}$, $\phi$ be a function satisfying $(\mathrm{H} 0)$, and $\lambda>0$ such that $\mu_{n, \phi_{\lambda}}$ is isotropic. There exists a universal constant $C>0$ such that

i) if $\phi$ satisfies (H1) then

$$
\forall a \in[0,1], \quad \operatorname{Is}_{\mu_{n, \phi_{\lambda}}}(a) \geq C \phi^{-1}(1) \frac{(a \wedge(1-a)) \log \frac{1}{a \wedge(1-a)}}{\phi^{-1}\left(\log \frac{1}{a \wedge(1-a)}\right)},
$$

ii) if $\phi$ satisfies $(\mathrm{H} 2(\alpha, \beta))$ then

$$
\forall a \in[0,1], \quad \operatorname{Is}_{\mu_{n, \phi_{\lambda}}}(a) \geq C(1 / \alpha)^{1 / \beta}(a \wedge(1-a)) \sqrt{\log \frac{1}{a \wedge(1-a)}} .
$$

These theorems are optimal, in a sense specified in Section 7. Note that a straightforward application of Bobkov's inequality for log-concave measures (Theorem 8) leads to isoperimetric inequalities with a (non-optimal) dependence in the dimension. For instance, Lemma 4 of [2] and the computation of exponential moments imply the Theorem 3 with $C / \sqrt{n}$ instead of $C$.

Let us finish this section by showing how Theorem 4 implies the two other ones. To deduce Theorem 5, we play with the properties of $\phi$ (see Lemma 6). Indeed, under (H1),

$$
\frac{\phi^{-1}(n)}{\sqrt{n}} \geq \phi^{-1}(1)
$$

and when $a \leq 1 / 2$,

$$
\phi^{-1}\left(\log \frac{1}{a}\right) \geq \frac{\phi^{-1}(\log 2)}{\sqrt{\log 2}} \sqrt{\log \frac{1}{a}} \geq \phi^{-1}(1) \sqrt{\log 2} \sqrt{\log \frac{1}{a}} .
$$

Hence, if $\phi$ satisfies (H1) and $a \in[0,1 / 2]$,

$$
J_{n, \phi}(a) \geq \phi^{-1}(1) \sqrt{\log 2} \frac{a \log \frac{1}{a}}{\phi^{-1}\left(\log \frac{1}{a}\right)} .
$$


Similarly, under $(\mathrm{H} 2)$ - all the more under $(\mathrm{H} 2(\alpha, \beta))-$,

$$
\forall a \leq e^{-n}, \quad \phi^{-1}\left(\log \frac{1}{a}\right) \leq \frac{\phi^{-1}(n)}{\sqrt{n}} \sqrt{\log \frac{1}{a}},
$$

therefore, for every $a \in[0,1 / 2]$,

$$
J_{n, \phi}(a) \geq a \sqrt{\log \frac{1}{a}} .
$$

As for Theorem 3, the second point of Theorem 4 gives us the result for $n$ large enough. On the other hand, the first point lead to the following isoperimetric inequality for $\mu_{n, \alpha}$ true for every $n$ :

$$
\forall a \in[0,1], \quad \operatorname{Is}_{\mu_{n, \alpha}}(a) \geq C n^{1 / 2-1 / \alpha} J_{n, \alpha}(a) .
$$

where $C>0$ is a universal constant. Here, the moments can be explicitly computed, and thus also $\lambda>0$ such that $\mu(d x)=Z^{-1} e^{-|\lambda x|^{\alpha}} d x$ is isotropic. We obtain

$$
\mathrm{E}_{\mu_{n, \alpha}}\left(|X|^{2}\right)=\frac{\Gamma((n+2) / \alpha)}{\Gamma(n / \alpha)} \quad \text { and } \quad \lambda=\sqrt{\frac{\Gamma((n+2) / \alpha)}{n \Gamma(n / \alpha)}} .
$$

Thanks to the formula of change of variable, one can deduce that for every $n \in \mathbb{N}^{*}$,

$$
\forall a \in[0,1], \quad \operatorname{Is}_{\mu}(a) \geq \lambda C n^{1 / 2-1 / \alpha} J_{n, \alpha}(a) .
$$

Finally, $\lambda n^{1 / 2-1 / \alpha} \geq c$ where $c>0$ is a universal constant because of the Stirling formula and the equivalent $\phi(x) \sim 1 / x$ at 0 . The second part of the theorem is deduced like above.

The next lemma sums up some properties of $\phi$ under our assumptions.

Lemma 6. Let $t \geq 1$ and $x \geq 0$.

- Under (H0), it holds

$$
\begin{aligned}
t \phi(x) & \leq \phi(t x), \\
\phi^{-1}(t x) & \leq t \phi^{-1}(x), \\
\phi(x) & \leq x \phi^{\prime}(x) .
\end{aligned}
$$

- Under (H1), it holds:

$$
\begin{aligned}
t \phi(x) & \leq \phi(t x) \leq t^{2} \phi(x), \\
\sqrt{t} \phi^{-1}(x) & \leq \phi^{-1}(t x) \leq t \phi^{-1}(x), \\
\phi(x) & \leq x \phi^{\prime}(x) \leq 2 \phi(x), \\
\phi^{\prime}(t x) & \leq 2 t \phi^{\prime}(x) .
\end{aligned}
$$


- Under (H2), it holds:

$$
\begin{aligned}
t^{2} \phi(x) & \leq \phi(t x), \\
\phi^{-1}(t x) & \leq \sqrt{t} \phi^{-1}(x), \\
2 \phi(x) & \leq x \phi^{\prime}(x) .
\end{aligned}
$$

- Under (H2'), it holds:

$$
\begin{aligned}
t^{\alpha} \phi(x) & \leq \phi(t x) \leq t^{2 \alpha} \phi(x), \\
t^{1 / 2 \alpha} \phi^{-1}(x) & \leq \phi^{-1}(t x) \leq t^{1 / \alpha} \phi^{-1}(x), \\
\alpha \phi(x) & \leq x \phi^{\prime}(x) \leq 2 \alpha \phi(x) .
\end{aligned}
$$

We establish in Section 2 the isoperimetric inequality for the radial measure. The proof relies on an inequality for log-concave measures due to Bobkov and some estimates of probabilities of balls. Section 3 is devoted to the argument of tensorization which yields the isoperimetric inequality on $\mathbb{R}^{n}$ from the ones for the radial measure and the uniform probability measure on the sphere. A cut-off argument is needed to get rid of the case of large radius. This tensorization relies on a functional version of the inequality, whose proof is postponed to Section 4 where we study the properties of the profile $J_{n, \phi}$. We combine the previous results in Section 5 to prove the first point of Theorem 4. Eventually, we discuss the isotropic case in Section 6 and the optimality of these inequalities in Section 7.

\section{Isoperimetry for the radial measure $\nu_{n, \phi}$}

In order to deal with $\mu_{n, \phi}$, a first step is to establish a similar isoperimetric inequality for its radial marginal. Recall that $\nu_{n, \phi}$ is the probability measure on $\mathbb{R}^{+}$of density proportional to $r^{n-1} e^{-\phi(r)}$.

Theorem 7. There exists a universal constant $C>0$ such that, for every $n \in \mathbb{N}^{*}$ and every function $\phi$ satisfying $(\mathrm{H} 1)$ or $(\mathrm{H} 2)$,

$$
\forall a \in[0,1 / 2], \quad \operatorname{Is}_{\nu_{n, \phi}}(a) \geq C \frac{\sqrt{n}}{\phi^{-1}(n)} J_{n, \phi}(a) .
$$

As $\nu_{n, \phi}$ is a log-concave measure, we can apply the isoperimetric inequality shown by Bobkov in [9].

Theorem 8 (Bobkov [9]). If $\mu$ is a log-concave measure on $\mathbb{R}^{n}$, then for every $r>0$ and every $x_{0} \in \mathbb{R}^{n}$,

$$
\forall a \in[0,1 / 2], \quad 2 r \operatorname{Is}_{\mu}(a) \geq a \log \frac{1}{a}+(1-a) \log \frac{1}{1-a}+\log \mu\left\{\left|x-x_{0}\right| \leq r\right\} .
$$


To derive other inequalities, one chooses $r$ as small as possible but with $\mu\left\{\left|x-x_{0}\right| \leq r\right\}$ large enough, such that the sum of the two last terms is non-negative. This requires explicit estimates of probabilities of balls. In our case, we will use two different estimates valid for two ranges of $r$, leading to inequalities for two ranges of $a$. The first lemma is due to Klartag [15]. The balls are centered at the maximum of the density in order to capture a large fraction of the mass.

Lemma 9 (Klartag [15]). Let $\nu(d r)=r^{n-1} \rho(r) d r$ be a probability measure on $\mathbb{R}^{+}$with $\rho$ a log-concave function of class $\mathcal{C}^{2}$. Let $r_{0}$ be the point where the density reaches its maximum. Then,

$$
\forall \delta \in[0,1], \quad \nu\left\{\left|r-r_{0}\right| \geq \delta r_{0}\right\} \leq C_{1} e^{-c_{1} n \delta^{2}}
$$

where $C_{1}>1$ and $0<c_{1}<1$ are universal constants.

Bobkov's inequality combined with the latter lemma leads to the following proposition.

Proposition 10. There exist two universal constants $0<c<1$ and $C>0$ such that for all functions $\phi$ satisfying $(\mathrm{H} 0)$ and all $n$ large enough to ensure $e^{-c n}<\frac{1}{2}$, it holds

$$
\forall a \in\left[e^{-c n}, \frac{1}{2}\right], \quad \operatorname{Is}_{\nu_{n, \phi}}(a) \geq C \frac{\sqrt{n}}{\phi^{-1}(n)} a \sqrt{\log \frac{1}{a}}
$$

Proof. Let $C_{1}$ and $c_{1}$ be the constants given by Lemma 9. Let $K>0$ and set

$$
\delta=\sqrt{\frac{K \log \frac{1}{a}}{c_{1} n}} .
$$

Choose $a \in\left[\exp \left(-\frac{c_{1} n}{K}\right), \frac{1}{2}\right]$ and $K>\frac{\log C_{1}}{\log 2}$. It follows that $\delta \leq 1$ and $1-C_{1} a^{K}>0$. Then Lemma 9 implies

$(1-a) \log \frac{1}{1-a}+\log \nu_{n, \phi}\left\{\left|r-r_{0}\right| \leq \delta r_{0}\right\} \geq(1-a) \log \frac{1}{1-a}+\log \left(1-C_{1} a^{K}\right)$.

The right-hand term of $(2.2)$ cancels at 0 and is concave in $a$ on $\left[0, \frac{1}{2}\right]$ if $K \geq 1$. Take $K$ large enough such that it is also non-negative at $\frac{1}{2}$. Thus, by concavity, it is non-negative on $\left[0, \frac{1}{2}\right]$. So Bobkov's formula (2.1) yields

$$
\operatorname{Is}_{\nu_{n, \phi}}(a) \geq \frac{1}{2} \sqrt{\frac{c_{1} n}{K r_{0}^{2}}} a \sqrt{\log \frac{1}{a}} .
$$


It remains to estimate the point $r_{0}$ where the density of $\nu_{n, \phi}$ reaches its maximum. The differentiation of the density leads to

$$
r_{0} \phi^{\prime}\left(r_{0}\right)=n-1
$$

By Lemma $6, \phi\left(r_{0}\right) \leq n-1$. Thus

$$
r_{0} \leq \phi^{-1}(n)
$$

To cope with smaller sets, we need another estimate for balls with greater radius.

Lemma 11. Let $\phi$ be a function satisfying $(\mathrm{H} 0)$ and $n \in \mathbb{N}^{*}$. Then for all $r \geq \phi^{-1}(2 n)$,

$$
\nu_{n, \phi}\{(r,+\infty)\} \leq F_{n, \phi}(r)=\left(\frac{e r}{\phi^{-1}(n)}\right)^{n} e^{-\phi(r)} \leq 1 .
$$

Note that this tail bound gives estimates of probability of balls centered at 0 for $\nu_{n, \phi}$, but also for $\mu_{n, \phi}$ since

$$
\nu_{n, \phi}\{(r,+\infty)\}=\mu_{n, \phi}\{|x| \geq r\} .
$$

This lemma can thereby be used to derive isoperimetric inequalities from Bobkov's formula for both measures.

Proof. The main tool is integration by part.

$$
\begin{aligned}
\int_{r}^{+\infty} t^{n-1} e^{-\phi(t)} d t & =\int_{r}^{+\infty} \frac{t^{n-1}}{\phi^{\prime}(t)} \phi^{\prime}(t) e^{-\phi(t)} d t \\
& =\frac{r^{n-1}}{\phi^{\prime}(r)} e^{-\phi(r)}+\int_{r}^{+\infty}\left[\frac{n-1}{t \phi^{\prime}(t)}-\frac{\phi^{\prime \prime}(t)}{\left(\phi^{\prime}(t)\right)^{2}}\right] t^{n-1} e^{-\phi(t)} d t \\
& \leq \frac{r^{n-1}}{\phi^{\prime}(r)} e^{-\phi(r)}+\int_{r}^{+\infty} \frac{n-1}{t \phi^{\prime}(t)} t^{n-1} e^{-\phi(t)} d t
\end{aligned}
$$

If $t \geq r \geq \phi^{-1}(2 n) \geq \phi^{-1}(2(n-1))$, then $t \phi^{\prime}(t) \geq 2(n-1)$. So the last integral in the above inequality is less than $\frac{1}{2} \int_{r}^{+\infty} t^{n-1} e^{-\phi(t)} d t$. Moreover $r \phi^{\prime}(r) \geq 2 n$. Hence

$$
\int_{r}^{+\infty} t^{n-1} e^{-\phi(t)} d t \leq 2 \frac{r^{n-1}}{\phi^{\prime}(r)} e^{-\phi(r)} \leq \frac{r^{n}}{n} e^{-\phi(r)}
$$


It remains to deal with the normalization constant which makes $\nu_{n, \phi}$ a probability measure:

$$
\begin{aligned}
\int_{0}^{+\infty} n t^{n-1} e^{-\phi(t)} d t & \geq \int_{0}^{\phi^{-1}(n)} n t^{n-1} e^{-\phi(t)} d t \\
& \geq e^{-n} \int_{0}^{\phi^{-1}(n)} n t^{n-1} d t=\left(\frac{\phi^{-1}(n)}{e}\right)^{n} .
\end{aligned}
$$

Putting all together, we get the desired bound on the tail of $\nu_{n, \phi}$ :

$$
\nu_{n, \phi}\{(r,+\infty)\}=\frac{\int_{r}^{+\infty} t^{n-1} e^{-\phi(t)} d t}{\int_{0}^{+\infty} t^{n-1} e^{-\phi(t)} d t} \leq\left(\frac{e r}{\phi^{-1}(n)}\right)^{n} e^{-\phi(r)}
$$

Then one can show that the bound is non-increasing for $r \geq \phi^{-1}(n)$ and is equal to 1 for $r=\phi^{-1}(n)$.

Then, we show an isoperimetric inequality simultaneously for $\mu_{n, \phi}$ and $\nu_{n, \phi}$ in the range of small sets.

Proposition 12. For every $c>0$, there exists $C>0$ such that for all functions $\phi$ satisfying the hypotheses of $(\mathrm{HO})$,

$$
\forall a \in\left[0, e^{-c n} \wedge \frac{1}{2}\right], \quad \operatorname{Is}_{\mu}(a) \geq C \frac{a \log \frac{1}{a}}{\phi^{-1}\left(\log \frac{1}{a}\right)},
$$

where $\mu$ stands for $\mu_{n, \phi}$ or $\nu_{n, \phi}$.

Proof. As before, we start from (2.1) and set $r(a)=\phi^{-1}\left(K \log \frac{1}{a}\right)$, where $K$ is a constant large enough to ensure

$$
\begin{gathered}
K c \geq 2, \\
K-1 \geq \frac{1}{c}, \\
e K c \exp (-(K-1) c) \leq \frac{1}{2} .
\end{gathered}
$$

By Lemma $6, r \leq K \phi^{-1}\left(\log \frac{1}{a}\right)$, as $K>1$. So the result is deduced from Bobkov's inequality (2.1) provided that

$$
(1-a) \log \frac{1}{1-a}+\log \mu_{n, \phi}\{|x| \leq r\} \geq 0 .
$$

Now, by concavity, for all $x \in\left[0, \frac{1}{2}\right]$,

$$
(1-x) \log \frac{1}{1-x} \geq \log 2 x, \quad \text { and } \quad \log (1-x) \geq-2 \log 2 x .
$$


So, for all $a \in\left[0, \frac{1}{2}\right]$,

$$
(1-a) \log \frac{1}{1-a}+\log \mu_{n, \phi}\{|x| \leq r\} \geq \log 2\left(a-2 F_{n, \phi}(r)\right) \geq 0,
$$

as soon as

$$
r \geq \phi^{-1}(2 n) \quad \text { and } \quad F_{n, \phi}(r) \leq \frac{a}{2}
$$

Assume that $a \leq \exp (-c n) \wedge \frac{1}{2}$. Then $r \geq \phi^{-1}(K c n) \geq \phi^{-1}(2 n)$ by $(2.3)$. Let us define the function $G$ by

$$
G(a)=\frac{F_{n, \phi}(r(a))}{a} .
$$

Then (2.6) holds as soon as $G(a) \leq \frac{1}{2}$. To handle this, it is easier to look on $G$ as a function of $r$. We know that $a=\exp \left(-\frac{\phi(r)}{K}\right)$. So

$$
G(a)=\left(\frac{e r}{\phi^{-1}(n)}\right)^{n} \exp \left(-\phi(r)\left(1-\frac{1}{K}\right)\right) .
$$

This function is non-increasing in $r$ when

$$
r \phi^{\prime}(r) \geq \frac{n}{1-\frac{1}{K}} .
$$

This is the case if $r \geq \phi^{-1}\left(\frac{K n}{K-1}\right)$. Moreover $\phi^{-1}(K c n) \geq \phi^{-1}\left(\frac{K n}{K-1}\right)$ by (2.4). Thus, when $a \leq \exp (-c n)$,

$$
G(a) \leq G(\exp (-c n)) \leq[e K c \exp (-(K-1) c)]^{n} \leq \frac{1}{2^{n}} \leq \frac{1}{2} .
$$

So far, we know that there exists a universal constant $C>0$ such that, for every function $\phi$ satisfying (H0),

$$
\forall a \in\left[0, e^{-n}\right] \cup\left[e^{-c n}, 1 / 2\right], \quad \operatorname{Is}_{\nu_{n, \phi}}(a) \geq C \frac{\sqrt{n}}{\phi^{-1}(n)} J_{n, \phi}(a),
$$

where $c \in(0,1)$ is the constant from Proposition 10. Now, if $a \in\left[e^{-n}, e^{-c n}\right]$, then $\log \frac{1}{a} \leq n$ and under $(\mathrm{H} 1)$,

$$
\phi^{-1}\left(\log \frac{1}{a}\right) \leq \frac{\phi^{-1}(n)}{\sqrt{n}} \sqrt{\log \frac{1}{a}} .
$$

Thus, Proposition 12 implies that under (H1),

$$
\forall a \in\left[e^{-n}, e^{-c n}\right], \quad \operatorname{Is}_{\nu_{n, \phi}}(a) \geq C \frac{\sqrt{n}}{\phi^{-1}(n)} a \sqrt{\log \frac{1}{a}},
$$

and Theorem 7 is shown under the hypothesis (H1). We could also derive the same inequality under (H2), but with $\sqrt{n} / \phi^{-1}(c n)$ instead of $\sqrt{n} / \phi^{-1}(n)$. So we prove it directly, following the proof of Proposition 12. 
Proposition 13. For every $c>0$, there exists $C>0$ such that for all functions $\phi$ satisfying $(\mathrm{H} 2)$,

$$
\forall a \in\left[0, e^{-c n} \wedge \frac{1}{2}\right], \quad \operatorname{Is}_{\mu}(a) \geq C \frac{\sqrt{n}}{\phi^{-1}(n)} a \sqrt{\log \frac{1}{a}},
$$

where $\mu$ stands for $\mu_{n, \phi}$ or $\nu_{n, \phi}$.

Proof. We set

$$
r(a)=\sqrt{\frac{K\left(\phi^{-1}(n)\right)^{2}}{n} \log \frac{1}{a},}
$$

where $K$ is a constant large enough to satisfy

$$
\begin{gathered}
K c \geq 2, \\
K-1 \geq \frac{1}{2 c}, \\
e \sqrt{K c} \exp (-(K-1) c) \leq \frac{1}{2} .
\end{gathered}
$$

Assume that $a \leq \exp (-c n) \wedge \frac{1}{2}$, then

$$
r \geq \sqrt{K c} \phi^{-1}(n) \geq \sqrt{\frac{K c}{2}} \phi^{-1}(2 n) \geq \phi^{-1}(2 n) .
$$

So we can use the estimate from Lemma 11. Consider as before

$$
G(a)=\frac{F_{n, \phi}(r(a))}{a} .
$$

Then, as explained in the proof of Proposition 12, Bobkov's formula (2.1) yields the required isoperimetric inequality as soon as

$$
G(a) \leq \frac{1}{2}
$$

From (2.7), we deduce

$$
a=\exp \left(-\frac{n r^{2}}{K\left(\phi^{-1}(n)\right)^{2}}\right) .
$$

So if we express $G$ as a function of $r$,

$$
G(a)=\left(\frac{e r}{\phi^{-1}(n)}\right)^{n} \exp \left(-\phi(r)+\frac{n r^{2}}{K\left(\phi^{-1}(n)\right)^{2}}\right) .
$$


The derivative $\partial_{r} G^{\frac{1}{n}}$ is of the same sign as

$$
1+\frac{2 r^{2}}{K\left(\phi^{-1}(n)\right)^{2}}-\frac{r \phi^{\prime}(r)}{n} .
$$

Under (H2), $r \phi^{\prime}(r) \geq 2 \phi(r) \geq 2 n\left(r / \phi^{-1}(n)\right)^{2}$ as soon as $r \geq \phi^{-1}(n)$. Thus, when $r \geq \sqrt{K c} \phi^{-1}(n)$,

$$
\begin{aligned}
1+\frac{2 r^{2}}{K\left(\phi^{-1}(n)\right)^{2}}-\frac{r \phi^{\prime}(r)}{n} & \leq 1+\frac{2 r^{2}}{\left(\phi^{-1}(n)\right)^{2}}\left(\frac{1}{K}-1\right) \\
& \leq 1+\frac{2 r^{2}}{\left(\phi^{-1}(n)\right)^{2}}\left(-\frac{1}{2 K c}\right) \leq 0 .
\end{aligned}
$$

So $G$ is non-increasing in $r$ whenever $r \geq \sqrt{K c} \phi^{-1}(n)$. For all $a \leq \exp (-c n)$, it follows

$$
\begin{aligned}
G(a) & \leq(e \sqrt{K c})^{n} \exp \left(c n-\phi\left(\sqrt{K c} \phi^{-1}(n)\right)\right) \\
& \leq[e \sqrt{K c} \exp (-(K-1) c)]^{n} \leq \frac{1}{2} .
\end{aligned}
$$

Here we use again $(\mathrm{H} 2)$ which ensures that $\phi\left(\sqrt{K c} \phi^{-1}(n)\right) \geq K c n$.

\section{Tensorization and cut-off argument}

We derive the isoperimetric inequality for $\mu_{n, \phi}$ by tensorization from the ones for the radial measure and the uniform probability measure on the sphere, following the idea of the proof by Bobkov of Theorem 1. For that purpose, we need a functional version of our isoperimetric inequality. It exists in our setting as explained in Section 4.

Let $\kappa>0$. Let $J:[0,1] \rightarrow \mathbb{R}^{+}$be a continuous concave function symmetric with respect to $1 / 2$, with $J(0)=J(1)=0$ and $J(1 / 2)>0$, and such that the following property holds : for any measure $\mu$ on $\mathbb{R}^{d}$ and constant $C \geq 0$, if

$$
\mathrm{Is}_{\mu} \geq C J
$$

then for all smooth functions $f: \mathbb{R}^{d} \rightarrow[0,1]$,

$$
\kappa J\left(\int f d \mu\right) \leq \int J(f) d \mu+\frac{1}{C} \int|\nabla f| d \mu .
$$

These properties are all satisfied when $J$ is the isoperimetric profile of the logistic ditribution or of the Gaussian one, with $\kappa=1$ (see [4] and references therein). Actually, we shall see in the next section that this is also the case for $J=J_{n, \phi}$, with some universal $\kappa>0$. 
Remark. Ideally, one would expect $\kappa=1$. For instance the latter inequality implies the former one and is tight for constant functions only in the case $\kappa=1$. However this does not matter here as we tensorize only once.

For such profiles $J$, we can show the following proposition.

Proposition 14. Let $\mu$ be a measure on $\mathbb{R}^{n}$ with radial measure $\nu$. Assume that there exists positive constants $C_{\nu}$ and $C_{\sigma_{n-1}}$ such that

$$
\mathrm{Is}_{\nu} \geq C_{\nu} J \quad \text { and } \quad \mathrm{Is}_{\sigma_{n-1}} \geq C_{\sigma_{n-1}} J .
$$

There exist $\kappa_{1}, \kappa_{2}>0$ depending only on $\kappa$ such that, for every $r_{2}>r_{1}>0$ and $a \in[0,1 / 2]$, whenever

$$
r_{2}-r_{1} \geq \frac{1}{C_{\nu} J\left(\frac{1}{2}\right)}
$$

and

$$
\kappa_{1} \nu\left\{\left[r_{1},+\infty\right)\right\} \leq a \leq \frac{1}{2}
$$

then

$$
\operatorname{Is}_{\mu}(a) \geq \kappa_{2} \min \left(C_{\nu}, \frac{C_{\sigma_{n-1}}}{r_{2}}\right) J(a) .
$$

The two conditions (3.1) and (3.1) arise in a cut-off argument needed in the proof. The former condition ensures that the derivative of the cut-off function is not too large; the latter that the error made by dropping large $r$, is negligible compared to the measure of the considered sets.

Proof. Let $f: \mathbb{R}^{n} \rightarrow[0,1]$ be a smooth function. We recall some facts on radial and spherical differentiation. If we define $g$ on $\mathbb{R}^{+} \times \mathbb{S}^{n-1}$ by $g(r, \theta)=f(r \theta)$, then the partial derivatives of $g$ can be computed as follows:

$$
\begin{aligned}
\partial_{r} g & =\langle\nabla f, \theta\rangle, \\
\nabla_{\theta} g & =r \Pi_{\theta^{\perp}}(\nabla f),
\end{aligned}
$$

where $\Pi_{\theta^{\perp}}$ is the orthogonal projection on $\theta^{\perp}$. Hence,

$$
\begin{gathered}
\nabla f=\partial_{r} g \theta+\frac{1}{r} \nabla_{\theta} g, \\
|\nabla f|^{2}=\left|\partial_{r} g\right|^{2}+\frac{1}{r^{2}}\left|\nabla_{\theta} g\right|^{2} .
\end{gathered}
$$

First, we apply the functional inequality for $\sigma_{n-1}$ to the function $F$ defined on $\mathbb{S}^{n-1}$ by

$$
F(\theta)=\int f(r \theta) d \nu(r)
$$


As $\int F d \sigma_{n-1}=\int f d \mu$, this yields

$$
\kappa J\left(\int f d \mu\right) \leq \int J(F) d \sigma_{n-1}+\frac{1}{C_{\sigma_{n-1}}} \int\left|\nabla_{\mathbb{S}^{n-1}} F\right| d \sigma_{n-1} .
$$

On one hand,

$$
\nabla_{\mathbb{S}^{n-1}} F(\theta)=\int r \Pi_{\theta^{\perp}}(\nabla f)(r \theta) d \nu(r) .
$$

On the other hand, we can use the inequality for $\nu$ to bound $J(F)$. Indeed, for all $\theta \in \mathbb{S}^{n-1}$,

$$
\kappa J(F(\theta)) \leq \int J(f(r \theta)) d \nu(r)+\frac{1}{C_{\nu}} \int\left|\partial_{r} f(r \theta)\right| d \nu(r) .
$$

Putting all together,

$$
\begin{aligned}
\kappa^{2} J\left(\int f d \mu\right) \leq & \int J(f) d \mu \\
& +\frac{1}{C_{\nu}} \int\left|\partial_{r} f\right| d \mu+\frac{\kappa}{C_{\sigma_{n-1}}} \int|x|\left|\Pi_{\theta^{\perp}}(\nabla f)\right| d \mu(x) .
\end{aligned}
$$

We would like to get $|x|$ out of the last integral. As it is not bounded, we use a cut-off argument similar to the one in Sodin's article [21], while simpler in our case. Heuristically, we use the fact that on "a set of large measure", $|x|$ is almost constant, close to its expectation for instance. Let us introduce a cut-off function $h(r \theta)=h_{1}(r)$ with

$$
h_{1}= \begin{cases}1 & \text { on }\left[0, r_{1}\right) \\ \frac{r_{2}-r}{r_{2}-r_{1}} & \text { on }\left[r_{1}, r_{2}\right] \\ 0 & \text { on }\left(r_{2},+\infty\right)\end{cases}
$$

with $0<r_{1}<r_{2}$ to be chosen later (typically of the same order as $\mathrm{E}_{\mu}|X|$ ). It holds

$$
\nabla(f h)=h \nabla f+f \nabla h
$$

thus

$$
\begin{aligned}
\left|\partial_{r}(f h)\right| & \leq\left|\partial_{r} f\right|+|| f||_{\infty}\left|\partial_{r} h\right|, \\
\left|\Pi_{\theta^{\perp}}(\nabla(f h))\right| & \leq h\left|\Pi_{\theta^{\perp}}(\nabla f)\right| .
\end{aligned}
$$

As $h=0$ if $|x|>r_{2}$,

$$
\int|x|\left|\Pi_{\theta^{\perp}}(\nabla(f h))\right| d \mu(x) \leq r_{2} \int\left|\Pi_{\theta^{\perp}}(\nabla f)\right| d \mu(x) .
$$


Besides, we can bound the derivative of $h$ so that

$$
\int\left|\partial_{r} h\right| d \mu \leq \frac{\nu\left(\left[r_{1}, r_{2}\right]\right)}{r_{2}-r_{1}}
$$

Finally, Inequality (3.3) applied to $f h$ yields

$$
\begin{aligned}
& \kappa^{2} J\left(\int f h d \mu\right)-\int J(f h) d \mu-\frac{\|f\|_{\infty} \nu\left(\left[r_{1}, r_{2}\right]\right)}{C_{\nu}\left(r_{2}-r_{1}\right)} \leq \\
& \leq \max \left(\frac{1}{C_{\nu}}, \frac{\kappa r_{2}}{C_{\sigma_{n-1}}}\right)\left(\int\left|\partial_{r} f\right|+\left|\Pi_{\theta^{\perp}}(\nabla f)\right| d \mu\right) \\
& \leq \sqrt{2} \max \left(\frac{1}{C_{\nu}}, \frac{\kappa r_{2}}{C_{\sigma_{n-1}}}\right) \int|\nabla f| d \mu .
\end{aligned}
$$

Hence we have almost the functional inequality for $f$ and $\mu$ with an additional term that we expect to be negligible. It is easier to look at functions approximating characteristic functions to go back from $f h$ to $f$ in the left hand term.

Let $A \subset \mathbb{R}^{n}$ be a closed set of measure $a \leq \frac{1}{2}$. Let $K>0$ and $t \in(0,1)$ constants to be chosen later. Assume the following constraints on $r_{1}, r_{2}$, and $a$ :

$$
\begin{gathered}
C_{\nu}\left(r_{2}-r_{1}\right) \geq K, \\
\nu\left\{\left[r_{1},+\infty\right)\right\} \leq t a .
\end{gathered}
$$

Then it holds

$$
\begin{aligned}
& \mu\left\{\mathbb{1}_{A} h=1\right\} \geq \mu(A \backslash\{h<1\}) \geq(1-t) a, \\
& \mu\left\{\mathbb{1}_{A} h>0\right\} \leq a \leq \frac{1}{2} .
\end{aligned}
$$

As $J$ is non-decreasing on $\left(0, \frac{1}{2}\right)$, concave, and $J(0)=0$,

$$
J\left(\int \mathbb{1}_{A} h d \mu\right) \geq J((1-t) a) \geq(1-t) J(a) .
$$

Besides $J$ cancels at 0 and 1 , and reaches its maximum at $\frac{1}{2}$, so

$$
\begin{aligned}
\int J\left(\mathbb{1}_{A} h\right) d \mu & \leq J\left(\frac{1}{2}\right) \mu\left\{0<\mathbb{1}_{A} h<1\right\} \\
& \leq J\left(\frac{1}{2}\right)\left(\mu\left\{\mathbb{1}_{A} h>0\right\}-\mu\left\{\mathbb{1}_{A} h=1\right\}\right) \\
& \leq J\left(\frac{1}{2}\right) \text { ta. }
\end{aligned}
$$


As for the third term of (3.4), it is bounded by

$$
\frac{\nu\left(\left[r_{1}, r_{2}\right]\right)}{C_{\nu}\left(r_{2}-r_{1}\right)} \leq \frac{t a}{K}
$$

For $\varepsilon>0$, we approximate $\mathbb{1}_{A}$ by a smooth function $f_{\varepsilon}: \mathbb{R}^{n} \rightarrow[0,1]$ with $f_{\varepsilon}=1$ on $A$ and $f_{\varepsilon}=0$ outside $A_{\varepsilon}$. Then we apply (3.4) to $f_{\varepsilon}$ and let $\varepsilon$ to 0 , taking advantage of the continuity of $J$ :

$$
\sqrt{2} \max \left(\frac{1}{C_{\nu}}, \frac{\kappa r_{2}}{C_{\sigma_{n-1}}}\right) \mu^{+}(\partial A) \geq \kappa^{2}(1-t) J(a)-\left(J\left(\frac{1}{2}\right)+\frac{1}{K}\right) t a .
$$

Now by concavity, $J(a) \geq 2 J\left(\frac{1}{2}\right) a$ on $\left[0, \frac{1}{2}\right]$. Hence

$$
\begin{aligned}
\sqrt{2} \max \left(\frac{1}{C_{\nu}}, \frac{\kappa r_{2}}{C_{\sigma_{n-1}}}\right) \mu^{+}(\partial A) & \geq\left(\kappa^{2}(1-t)-\frac{J\left(\frac{1}{2}\right)+\frac{1}{K}}{2 J\left(\frac{1}{2}\right)} t\right) J(a) \\
& =\left(\kappa^{2}-t\left(\kappa^{2}+\frac{1}{2}+\frac{1}{2 K J\left(\frac{1}{2}\right)}\right)\right) J(a) .
\end{aligned}
$$

Taking for instance $K=\left(J\left(\frac{1}{2}\right)\right)^{-1}$ and $t=\kappa^{2} /\left(2\left(\kappa^{2}+1\right)\right)$ yields a non-trivial result.

Note that looking at closed sets was not a real restriction. Indeed, if $\liminf _{\varepsilon \rightarrow 0^{+}} \mu\left(A_{\varepsilon}\right)-\mu(A)>0$ then $\mu^{+}(\partial A)=+\infty$.

\section{Getting functional inequalities}

To apply Proposition 14 to our case, we need to know how to pass from an isoperimetric inequality to a functional inequality. When $\phi$ satisfies $(\mathrm{H} 1)$, we show that $J_{n, \phi}$ satisfies the required properties. Recall that $L_{2}(a)=$ $a \sqrt{\log \frac{1}{a}}$.

Lemma 15. Let $n \in \mathbb{N}^{*}$ and $\phi$ be a function satisfying (H1).

i) The function $J_{n, \phi}$ is continuous and concave on $[0,1]$, symmetric with respect to $1 / 2$, with $J_{n, \phi}(0)=J_{n, \phi}(1)=0$ and $J_{n, \phi}(1 / 2) \geq 1 / 2$.

ii) There exists an even log-concave probability measure on the real line $m_{n, \phi}$ such that $J_{n, \phi}=\mathrm{Is}_{m_{n, \phi}}$.

iii) There exists a universal constant $D>0$ such that

$$
\forall 0<t \leq s \leq \frac{1}{2}, \quad \frac{J_{n, \phi}(t)}{L_{2}(t)} \leq D \frac{J_{n, \phi}(s)}{L_{2}(s)}
$$


iv) Let $\mu$ be a measure on $\mathbb{R}^{d}$ and $C>0$. If

$$
\mathrm{Is}_{\mu} \geq C J_{n, \phi},
$$

then for all smooth functions $f: \mathbb{R}^{d} \rightarrow[0,1]$,

$$
\kappa J_{n, \phi}\left(\int f d \mu\right) \leq \int J_{n, \phi}(f) d \mu+\frac{1}{C} \int|\nabla f| d \mu,
$$

where $\kappa>0$ is a universal constant.

Proof. For the first point, we only need to prove the concavity. Assume that $e^{-n} \leq 1 / 2$. It is easy to see that $J_{n, \phi}$ is concave on $\left[0, e^{-n}\right]$ and $\left[e^{-n}, 1 / 2\right]$, and non-decreasing on $[0,1 / 2]$. It remains to compare the left and right derivative of $J_{n, \phi}$ at point $e^{-n}$. Under (H1),

$$
\forall x>0, \quad \phi^{\prime} \circ \phi^{-1}(x) \leq 2 \frac{x}{\phi^{-1}(x)},
$$

thus

$$
\left[\frac{x}{\phi^{-1}(x)}\right]^{\prime}=\frac{1}{\phi^{-1}(x)}\left(1-\frac{x}{\phi^{-1}(x) \phi^{\prime} \circ \phi^{-1}(x)}\right) \leq \frac{1}{2 \phi^{-1}(x)} .
$$

It follows

Moreover,

$$
\left[\frac{a \log \frac{1}{a}}{\phi^{-1}\left(\log \frac{1}{a}\right)}\right]^{\prime} \geq \frac{\log \frac{1}{a}-1 / 2}{\phi^{-1}\left(\log \frac{1}{a}\right)}
$$

$$
\left[a \sqrt{\log \frac{1}{a}}\right]^{\prime}=\frac{\log \frac{1}{a}-1 / 2}{\phi^{-1}\left(\log \frac{1}{a}\right)} .
$$

So, denoting respectively by $\partial_{-} J_{n, \phi}$ and $\partial_{+} J_{n, \phi}$ the left and right derivatives of $J_{n, \phi}$, it holds

$$
\partial_{-} J_{n, \phi}\left(e^{-n}\right) \geq \partial_{+} J_{n, \phi}\left(e^{-n}\right) .
$$

This shows the concavity of $J_{n, \phi}$. The second point is a direct consequence of $i$ ), according to Bobkov [8]. The point iii) holds for $t \leq s \leq e^{-n}$ as the function defined by

$$
t \mapsto \frac{L_{\phi}(t)}{L_{2}(t)}=\frac{\sqrt{\log \frac{1}{t}}}{\phi^{-1}\left(\log \frac{1}{t}\right)}
$$

is non-decreasing under (H1). When $e^{-n} \leq t \leq s$, the quotient is constant equal to 1 . If $t \leq e^{-n} \leq s$, we just use the transitivity of such inequalities.

Let us turn now to the last point of the lemma. As $m_{n, \phi}$ is an even logconcave probability measure on the real line, half-lines solve the associated isoperimetric problem (see e.g. [8]). So, if $f_{\phi}: x \mapsto \frac{e^{-\phi(|x|)}}{Z_{\phi}}$ is the density of $m_{n, \phi}$ and $F_{\phi}(x)=m_{n, \phi}\{(-\infty, x)\}$ its cumulative distribution function, then

$$
J_{n, \phi}=\mathrm{Is}_{m_{n, \phi}}=f_{\phi} \circ F_{\phi}^{-1} .
$$


Thereby the transfer principle emphasized by Barthe in [2] holds: if $\mathrm{Is}_{\mu} \geq c J_{n, \phi}$ then $\mu$ satisfies essentially the same functional inequalities as $m_{n, \phi}$. As a consequence, it remains to establish that for all smooth functions $f: \mathbb{R} \rightarrow[0,1]$

$$
\kappa J_{n, \phi}\left(\int f d m_{n, \phi}\right) \leq \int J_{n, \phi}(f) d m_{n, \phi}+\int\left|f^{\prime}\right| d m_{n, \phi} .
$$

Now, applying the 2-dimensional isoperimetric inequality to the set

$$
\left\{(x, y) \in \mathbb{R}^{2} ; y \leq F_{\phi}^{-1}(f(x))\right\},
$$

one can show (see e.g. [3]) that

$$
\mathrm{Is}_{m_{n, \phi}}^{\otimes 2}\left(\int f d m_{n, \phi}\right) \leq \int J_{n, \phi}(f) d m_{n, \phi}+\int\left|f^{\prime}\right| d m_{n, \phi} .
$$

So, $i v)$ is shown if there exists a universal $\kappa>0$ such that

$$
\mathrm{Is}_{m_{n, \phi} \otimes 2} \geq \kappa J_{n, \phi}
$$

Actually, the following stronger dimension-free inequality holds.

Lemma 16. There exists $\kappa>0$ such that for every $\phi$ satisfying (H1) and all $n$,

$$
\mathrm{Is}_{m_{n, \phi} \otimes n} \geq \kappa J_{n, \phi}
$$

To prove it, we check the simple criterion given by E. Milman in [19]: " $J_{n, \phi}$ does not violate the Central-Limit obstruction with rate $D$ ". This is Lemma 15-iii).

\section{Isoperimetry for $\mu_{n, \phi}$}

Now we can apply Proposition 14 to $\mu_{n, \phi}$ with $J=J_{n, \phi}$ when $\phi$ satisfies (H1) or $J=L_{2}$ when $\phi$ satisfies (H2). Indeed, $J_{n, \phi}$ satisfies the required properties under (H1) according to the previous section, and $L_{2}$ also since $L_{2}=J_{n, \phi_{2}}$ with $\phi_{2}: x \mapsto x^{2}$ satisfying (H1). Moreover,

$$
\mathrm{Is}_{\nu_{n, \phi}} \geq C_{\nu_{n, \phi}} J
$$

with $C_{\nu_{n, \phi}}=C \frac{\sqrt{n}}{\phi^{-1}(n)}$, where $C>0$ is a universal constant, by Theorem 7 . This is direct under (H1). If $\phi$ satisfies (H2), we use that $J_{n, \phi} \geq L_{2}$ as explained page 98 . 
As for the sphere, it is known that $\sigma_{n-1}$ satisfies the Gaussian isoperimetry with a constant of order $\sqrt{n}$, e.g. by a curvature-dimension criterion (cf. [1]). That means that for every $a \leq \frac{1}{2}$, and every $\phi$ satisfying (H1),

$$
\operatorname{Is}_{\sigma_{n-1}}(a) \geq C \sqrt{n} L_{2}(a) .
$$

As $L_{2} \geq J_{n, \phi}$ when $\phi$ satisfies (H1), we conclude that, under (H1) or (H2),

$$
\operatorname{Is}_{\sigma_{n-1}}(a) \geq C \sqrt{n} J(a) .
$$

Proposition 17. Let $n \in \mathbb{N}^{*}$ and $\phi$ be a function satisfying (H1) or (H2). If $e^{-n}<\frac{1}{2}$, then there exists a universal constant $C>0$ such that

$$
\forall a \in\left[e^{-n}, \frac{1}{2}\right], \quad \operatorname{Is}_{\mu_{n, \phi}}(a) \geq C \frac{\sqrt{n}}{\phi^{-1}(n)} a \sqrt{\log \frac{1}{a}} .
$$

Proof. Let $\kappa$ be the constant coming from Lemma 15-iv) for $J_{n, \phi}$ or $J_{n, \phi_{2}}$ if $\phi$ satisfies (H1) or (H2) respectively. Then let $\kappa_{1}$ and $\kappa_{2}$ be the corresponding constants given by Proposition 14. Set $c$ large enough to ensure

$$
\begin{gathered}
c \geq 2, \\
\max \left(\kappa_{1}, 1\right) e c e^{-c} \leq e^{-1} .
\end{gathered}
$$

If we take $r_{1}=\phi^{-1}(c n)$, then we know by Lemma 11 that

$$
\kappa_{1} \nu_{n, \phi}\left\{\left(r_{1},+\infty\right)\right\} \leq \kappa_{1}\left[e c e^{-c}\right]^{n} \leq e^{-n} .
$$

Here we use that $\phi^{-1}(c n) \leq c \phi^{-1}(n)$. So for every $\phi, n$, and $a \in\left[e^{-n}, \frac{1}{2}\right]$, the condition (3.2) holds, i.e.

$$
\kappa_{1} \nu_{n, \phi}\left\{\left[r_{1},+\infty\right)\right\} \leq a \leq \frac{1}{2}
$$

Now there exists a universal $C>0$ such that $C_{\nu_{n, \phi}} \geq C_{\frac{\sqrt{n}}{\phi^{-1}(n)}}$ as explained at the beginning of the section. So, if we set $r_{2}=\left(1+\frac{2}{C}\right) \phi^{-1}(c n)$, then the condition (3.1) is also satisfied, i.e.

$$
r_{2}-r_{1} \geq \frac{2}{C_{\nu_{n, \phi}}} \geq \frac{1}{C_{\nu_{n, \phi}} J\left(\frac{1}{2}\right)} .
$$

Thus Proposition 14 yields

$$
\operatorname{Is}_{\mu_{n, \phi}}(a) \geq \kappa_{2} \min \left(C_{\nu_{n, \phi}}, \frac{C_{\sigma_{n-1}}}{r_{2}}\right) J(a) .
$$


Now $J(a)=a \sqrt{\log \frac{1}{a}}$ when $a \in\left[e^{-n}, 1 / 2\right]$ and $r_{2} \leq c\left(1+\frac{2}{C}\right) \phi^{-1}(n)$. Finally, we have established

$$
\operatorname{Is}_{\mu_{n, \phi}}(a) \geq \kappa_{2} C \min \left(1,\left[c\left(1+\frac{2}{C}\right)\right]^{-1}\right) \frac{\sqrt{n}}{\phi^{-1}(n)} a \sqrt{\log \frac{1}{a}} .
$$

Therefore the first point of Theorem 4 is proved at least for $a$ large enough. We complete its proof with Proposition 12 for smaller sets.

\section{The isotropic case}

If we set $\phi_{\lambda}(x)=\phi(\lambda x)$ with $\lambda>0$, then one can see that

$$
\mathrm{Is}_{\mu_{n, \phi_{\lambda}}}=\lambda \mathrm{Is}_{\mu_{n, \phi}} .
$$

If we want $\mu_{n, \phi_{\lambda}}$ to be isotropic, then we have to choose

$$
\lambda=\sqrt{\frac{\mathrm{E}_{\mu_{n, \phi}}\left(|X|^{2}\right)}{n}} .
$$

So, to finish the proof of Theorem 4, it remains to show that $\mathrm{E}_{\mu_{n, \phi}}\left(|X|^{2}\right) \simeq$ $\left(\phi^{-1}(n)\right)^{2}$. Actually, we only need that $\mathrm{E}_{\mu_{n, \phi}}\left(|X|^{2}\right) \geq c\left(\phi^{-1}(n)\right)^{2}$. Undoubtedly, this must be quite standard, nevertheless we state and prove the next lemma for completeness.

Lemma 18. i) Let $\phi$ be a function satisfying $(\mathrm{H} 0)$. Define $r_{n}(\phi)$ the point where the density of the radial measure $\nu_{n, \phi}$ reaches its maximum, and $\mathrm{E}_{\mu_{n, \phi}}|X|^{2}$ the second moment of $\mu_{n \phi}$. For every $M>1$, there exists $n_{0} \in \mathbb{N}$ not depending on $\phi$ such that, for all $n \geq n_{0}$,

$$
\frac{1}{M} \sqrt{\mathrm{E}_{\mu_{n, \phi}}|X|^{2}} \leq r_{n}(\phi) \leq M \sqrt{\mathrm{E}_{\mu_{n, \phi}}|X|^{2}}
$$

ii) Moreover, if there exists $\alpha \geq \beta \geq 1$ such that $x \mapsto \phi(x) / x^{\alpha}$ is nonincreasing and $x \mapsto \phi(x) / x^{\beta}$ is non-decreasing, then

$$
\phi^{-1}(n) \geq r_{n}(\phi) \geq\left(\frac{1}{2 \alpha}\right)^{1 / \beta} \phi^{-1}(n) \geq\left(e^{-2 / e}\right)^{\alpha / \beta} \phi^{-1}(n) .
$$

Proof. To prove the first point, we can assume that $\mu=\mu_{n, \phi}$ is isotropic, that is to say that $\mathrm{E}_{\mu}\left(|X|^{2}\right)=n$. Let $X$ be a random variable with distribution $\mu$. In the following, we denote by $\mathbb{P}, \mathrm{E}$, and Var the corresponding 
probability, esperance, and variance. Let $\delta \in(0,1)$. In view of Lemma 9, there exist universal constants $c>0$ and $C>0$ such that

$$
\mathbb{P}\left\{\left|r_{n}(\phi)-\right| X|| \geq \delta r_{n}(\phi)\right\} \leq C e^{-c n \delta^{2}} .
$$

On the other hand, $\mu$ satisfies a Cheeger inequality with a universal constant, according to Theorem 2. Thus, Lipschitz functions concentrate exponentially (cf. $[12,17]$ ) and there exists a universal constant $d>0$ such that

$$
\forall t>0, \quad \mathbb{P}(|| X|-\mathrm{E}| X|| \geq t) \leq e^{-2 d t} .
$$

Moreover, by Jensen inequality,

$$
(\mathrm{E}|X|)^{2} \leq \mathrm{E}|X|^{2}=n,
$$

and Bobkov proved in [10] the following upper bound for the variance of $|X|$ to establish Theorem 1:

$$
\operatorname{Var}|X| \leq \frac{(\mathrm{E}|X|)^{2}}{n}
$$

which translates into

$$
(\mathrm{E}|X|)^{2} \geq \frac{n}{n+1} \mathrm{E}|X|^{2} \geq n-1 .
$$

Therefore,

$$
\sqrt{n} \geq \mathrm{E}|X| \geq \sqrt{n-1} \geq \sqrt{n}-\frac{1}{\sqrt{n}}
$$

From this estimation and the above concentration inequality, we deduce

$$
\forall h \geq \frac{2}{n}, \quad \mathbb{P}\left(\left|\frac{|X|}{\sqrt{n}}-1\right| \geq h\right) \leq e^{-d h \sqrt{n}} .
$$

Fix $\delta \in(0,1)$, and choose $n$ large enough to ensure $C e^{-c n \delta^{2}}+e^{-d \delta \sqrt{n}}<1$. Then there exists $x>0$ such that $\left|r_{n}(\phi)-x\right| \leq \delta r_{n}(\phi)$ and $|x-\sqrt{n}| \leq \delta \sqrt{n}$. It follows

$$
\frac{1-\delta}{1+\delta} \sqrt{n} \leq r_{n}(\phi) \leq \frac{1+\delta}{1-\delta} \sqrt{n}
$$

Now, $r_{n}(\phi)$ satisfies $r_{n}(\phi) \phi^{\prime}\left(r_{n}(\phi)\right)=n-1$. Therefore, as already mentioned, (H0) ensures that $r_{n}(\phi) \leq \phi^{-1}(n)$. Assume moreover the existence of $\alpha \geq \beta \geq 1$ such that $x \mapsto \phi(x) / x^{\alpha}$ is non-increasing and $x \mapsto \phi(x) / x^{\beta}$ is non-decreasing. Then

$$
r_{n}(\phi) \geq \phi^{-1}\left(\frac{n-1}{\alpha}\right) \geq \phi^{-1}\left(\frac{n}{2 \alpha}\right) \geq\left(\frac{1}{2 \alpha}\right)^{1 / \beta} \phi^{-1}(n) \geq\left(e^{-2 / e}\right)^{\alpha / \beta} \phi^{-1}(n) .
$$




\section{Optimality}

The inequalities of Theorem 4 are optimal, at least for a large range of $a$. Actually we can exhibit almost isoperimetric sets, i.e. sets whose boundary measure is minimal, up to a multiplicative constant. When $a$ is large, these are half-spaces which are the exact isoperimetric sets for the Gaussian measure. This is due to the fact that marginals of spherically symmetric log-concave measures are approximately Gaussian in high dimension. When $a$ is small, these are the complementary of balls. This is quite surprising since it holds also for the Gaussian measure. These two facts are stated in the next lemmas.

Lemma 19. Let $\phi$ be a function satisfying (H0) and $n \geq 4$. Let us choose $\lambda>0$ such that $\mu_{n, \phi_{\lambda}}$ is isotropic. Then, for every $r$ such that

$$
c_{1} \leq r \leq c_{2} n^{1 / 8}
$$

or every $a:=\mu_{n, \phi_{\lambda}}\left(\left\{x_{1} \geq r\right\}\right)$ such that

$$
e^{-c_{3} n^{1 / 16}} \leq a \leq e^{-c_{4}}<1 / 2
$$

it holds

$$
\mu_{n, \phi_{\lambda}}^{+}\left(\partial\left\{x_{1} \geq r\right\}\right) \leq C a \sqrt{\log \frac{1}{a}}
$$

where $C, c_{1}, \ldots, c_{4}>0$ are universal constants.

Lemma 20. Let $\phi$ be a function satisfying (H0). Assume moreover that there exists $\alpha \geq 1$ such that $x \mapsto \phi(x) / x^{\alpha}$ is non-increasing, and that $\phi$ is log-concave. Then there exists a universal constant $C>0$ such that, for every $n \geq \alpha+1$, whenever $a:=\mu_{n, \phi}\{|x| \geq r\} \leq e^{-3 n} \wedge 1 / 2$,

$$
\mu_{n, \phi}^{+}(\partial\{|x| \geq r\}) \leq C \alpha \frac{a \log \frac{1}{a}}{\phi^{-1}\left(\log \frac{1}{a}\right)} .
$$

Note that Theorem 5 gives also optimal lower bounds for $\mathrm{Is}_{\mu_{n, \phi}}$ among functions of the form $f(n) g(a)$. When $\phi$ satisfies (H1), the optimality in $a$ comes from the case $n=1$. In this case, we know that half-lines solve the isoperimetric problem and one can prove (see [6] or [13] for universal constants $c_{1}$ and $c_{2}$ ) that if $\phi$ satisfies $(\mathrm{H} 0)$ and $\sqrt{\phi}$ is concave - which implies $(\mathrm{H} 1)-$, then there exist $c_{1}, c_{2}>0$ such that

$$
c_{1} L_{\phi} \leq \mathrm{Is}_{\mu_{1, \phi}} \leq c_{2} L_{\phi}
$$


Beyond the Gaussian regime, the central limit theorem for convex bodies of Klartag (see [15]), in the simpler case of spherically symmetric distributions, ensures that we cannot find a profile bounding from below $\mathrm{Is}_{\mu_{n, \phi}}$ for all $n$, better than the Gaussian one (times a constant depending possibly on $n$ ). Else we should have concentration properties stronger than Gaussian. However by Klartag's theorem, there exists a sequence of positive number $\varepsilon_{n} \rightarrow 0$ such that for every Borel set $A \subset \mathbb{R}$ and every $r>0$,

$$
1-\mu_{n, \phi}\left(\left(A \times \mathbb{R}^{n-1}\right)_{r}\right) \geq 1-\gamma\left(A_{r}\right)-\varepsilon_{n},
$$

where $\gamma$ denotes the standard normal distribution. Thus we cannot have a rate of concentration valid for all $n$ and better than the Gaussian one. Now Poincaré inequalities are equivalent up to universal constants to Cheeger inequalities (see [20]), so the optimal constant in $n$ must in fact be independent of $n$ in the isotropic case, in view of Theorem 1 .

Let us now turn to the proofs of the two previous lemmas.

Proof of Lemma 19. The constants $c, C, c_{i}, \ldots$ denote here positive universal constants that may change from line to line. The main ideas are classical and related to the central limit theorem for convex sets: if $X$ follows the law $\mu_{n, \phi_{\lambda}}$, its radius concentrates around $\sqrt{n}$ and $\theta=X /|X|$ is a uniform variable on the sphere. Now the projection of $\sqrt{n} \theta$ on a line is known to tend towards a standard Gaussian. Then we deduce a bound for the boundary measure of a half-space.

Let us recall the concentration inequality (see page 114) proved under $(\mathrm{H} 0)$ :

$$
\forall h \geq \frac{2}{n}, \quad \mathbb{P}\left(\left|\frac{|X|}{\sqrt{n}}-1\right| \geq h\right) \leq e^{-c h \sqrt{n}} .
$$

On the other hand, if $\theta$ is a uniform variable on the sphere $\mathbb{S}^{n-1}$, the density of $\sqrt{n} \theta_{1}$ is

$$
C_{n} \mathbb{1}_{[-\sqrt{n}, \sqrt{n}]}(x)\left(1-\frac{x^{2}}{n}\right)^{(n-3) / 2}
$$

where $C_{n} \sim(2 \pi)^{-n / 2}$. Moreover, for every $x \in[0, \sqrt{n}]$, it holds

$$
\left(1-\frac{x^{2}}{n}\right)^{(n-3) / 2} \leq \exp \left(-\frac{x^{2}}{2}(1-3 / n)\right)
$$

so that, for $0 \leq r \leq R$,

$$
\mathbb{P}\left(\sqrt{n} \theta_{1} \in[r, R]\right) \leq C \gamma_{1}([r \sqrt{1-3 / n}, R \sqrt{1-3 / n}]) .
$$


Conversely, if $0 \leq x \leq R \leq \sqrt{n / 2}$, then

$$
\left(1-\frac{x^{2}}{n}\right)^{(n-3) / 2} \geq \exp \left(-\frac{x^{2}}{2}\left(1+R^{2} / n\right)\right) .
$$

To see that, we used that if $0 \leq u \leq U \leq 1 / 2$, then

$$
\log (1-u) \geq-u \frac{\log (1-U)}{-U} \geq-u \frac{-U-U^{2}}{-U}=-u(1+U)
$$

Hence, for $0 \leq r \leq R \leq \sqrt{n / 2}$,

$$
\mathbb{P}\left(\sqrt{n} \theta_{1} \in[r, R]\right) \geq C \exp \left(-\frac{R^{4}}{2 n}\right) \gamma_{1}([r, R])
$$

The following bounds on Gaussian tail will also be useful. One can show by integration by parts that for every $r>0$,

$$
\frac{e^{-r^{2} / 2}}{\sqrt{2 \pi} r}\left(1-\frac{1}{r^{2}}\right) \leq \gamma_{1}((r,+\infty)) \leq \frac{e^{-r^{2} / 2}}{\sqrt{2 \pi} r}
$$

We turn now to the proof of the bound. Let $X$ be of law $\mu_{n, \phi_{\lambda}}$. Let $r$ satisfy

$$
1<c_{1} \leq r \leq c_{2} n^{1 / 8}
$$

We choose $c_{2}$ small enough such that

$$
\frac{1}{6 r^{2}} \leq n / 2
$$

and

$$
6 r^{4} \leq c \sqrt{n}
$$

so that we can use (7.1) with $h=1 / 6 r^{2}$ and $e^{-c h \sqrt{n}} \leq e^{-r^{2} / 2}$. Thus, with probability $1-\exp \left(-r^{2} / 2\right)$, the random variables $\frac{|X|}{\sqrt{n}}, \frac{\sqrt{n}}{|X|}$ and $\frac{n}{|X|^{2}}$ all belong to $\left[1-1 / r^{2}, 1+1 / r^{2}\right]$. We choose also $c_{1}$ large enough to ensures

$$
e^{-3 / 2} \frac{1-c_{1}^{-2}}{1+c_{1}^{-2}}-\frac{e^{-c_{1}^{2} / 2}}{\sqrt{2}}>0 .
$$

Then, using the previous inequalities and that $t e^{-t^{2} r^{2} / 2} \leq C$ for every $t \geq 0$ and $r \geq 1$, and splitting the expectation according to the values of $|X|$, 
we get:

$$
\begin{aligned}
\mu^{+}\left(\partial\left\{x_{1} \geq r\right\}\right) & =\lim _{\varepsilon \rightarrow 0^{+}} \frac{1}{\varepsilon} \mu\left(\left\{x_{1} \in[r-\varepsilon, r]\right\}\right) \\
& =\lim _{\varepsilon \rightarrow 0^{+}} \frac{1}{\varepsilon} \mathrm{E}_{X}\left(\mathbb{P}\left(\sqrt{n} \theta_{1} \in\left[\frac{r-\varepsilon}{|X| / \sqrt{n}}, \frac{r}{|X| / \sqrt{n}}\right]\right)\right) \\
& \leq C \mathrm{E}_{X}\left(\lim _{\varepsilon \rightarrow 0^{+}} \frac{1}{\varepsilon} \gamma_{1}\left(\left[\frac{r-\varepsilon}{|X| / \sqrt{n-3}}, \frac{r}{|X| / \sqrt{n-3}}\right]\right)\right) \\
& \leq C \mathrm{E}_{X}\left(\frac{\sqrt{n-3}}{|X|} \exp \left(-\frac{(n-3)}{|X|^{2}} \frac{r^{2}}{2}\right)\right) \\
& \leq C\left(1+1 / r^{2}\right) \exp \left(-\frac{r^{2}}{2}\left(1-1 / r^{2}\right)(1-3 / n)\right) \\
& \quad+C \mathbb{P}\left(\frac{\sqrt{n}}{|X|} \text { and } \frac{n}{|X|^{2}} \notin\left[1-1 / r^{2}, 1+1 / r^{2}\right]\right) \\
& \leq C e^{-r^{2} / 2} .
\end{aligned}
$$

To bound from below the measure of a half-space, we use that $|X|$ is independent of $\theta=X /|X|$ and the bounds on the Gaussian tails:

$$
\begin{aligned}
a:= & \mu\left(\left\{x_{1} \geq r\right\}\right) \\
& \geq \mathbb{P}\left(\frac{\sqrt{n}}{|X|} \in\left[1-1 / r^{2}, 1+1 / r^{2}\right]\right) \mathbb{P}\left(\sqrt{n} \theta_{1} \in\left[r\left(1+1 / r^{2}\right), \sqrt{2} r\right]\right) \\
& \geq c \gamma_{1}\left(\left[r\left(1+1 / r^{2}\right), \sqrt{2} r\right]\right) \\
& \geq c\left(\frac{\exp \left(-r^{2}\left(1+r^{-2}\right)^{2} / 2\right)}{\sqrt{2 \pi} r\left(1+r^{-2}\right)}\left(1-\frac{1}{r^{2}}\right)-\frac{\exp \left(-r^{2}\right)}{2 \sqrt{\pi} r}\right) \\
& \geq c \frac{e^{-r^{2} / 2}}{\sqrt{2 \pi} r}\left(e^{-3 / 2} \frac{1-r^{-2}}{1+r^{-2}}-\frac{e^{-r^{2} / 2}}{\sqrt{2}}\right) \\
& \geq c \frac{e^{-r^{2} / 2}}{r} .
\end{aligned}
$$

These two inequalities lead to

$$
\mu^{+}\left(\partial\left\{x_{1} \geq r\right\}\right) \leq C a r .
$$

To estimate $r$, we bound from above $a$. We use the same techniques as for the lower bound to get:

$$
\begin{aligned}
a & \leq \mathbb{P}\left(\frac{\sqrt{n}}{|X|} \notin\left[1-1 / r^{2}, 1+1 / r^{2}\right]\right)+\mathbb{P}\left(\sqrt{n} \theta_{1} \in\left[r\left(1-1 / r^{2}\right),+\infty\right)\right) \\
& \leq e^{-r^{2} / 2}+C \gamma_{1}\left(\left[r\left(1-1 / r^{2}\right),+\infty\right)\right) \\
& \leq C e^{-r^{2} / 2}
\end{aligned}
$$


and

$$
r \leq C \sqrt{\log \frac{1}{a}}
$$

Therefore, we obtain

$$
\mu^{+}\left(\partial\left\{x_{1} \geq r\right\}\right) \leq C a \sqrt{\log \frac{1}{a}} .
$$

Finally, let us show that the conditions on $a$ imply indeed $c_{1} \leq r \leq c_{2} n^{1 / 8}$. Assume that

$$
e^{-c_{3} n^{1 / 16}} \leq a \leq e^{-c_{4}}<1 / 2 .
$$

Using (7.1) with a constant $h<1$ depending neither on $r$ nor $n$, we get

$$
a \leq e^{-c \sqrt{n}}+C \gamma_{1}((c r,+\infty)) \leq \frac{a}{2}+C \frac{e^{-c r^{2}}}{r}
$$

thus

$$
e^{-c_{3} n^{1 / 16}} \leq \frac{e^{-c r^{2}}}{r} \quad \text { and } \quad r \leq C n^{1 / 8}
$$

Conversely,

$$
a \geq\left(1-e^{-c \sqrt{n}}\right) e^{-C r^{4} / n} \gamma_{1}((C r,+\infty)) \geq c \gamma_{1}((C r,+\infty)),
$$

and so $r \geq c$.

Proof of Lemma 20. The same integration by parts as in Lemma 11 gives a lower bound for $a$. Indeed, under our hypotheses,

$$
\frac{\phi^{\prime \prime}(t)}{\phi^{\prime}(t)^{2}} \leq \frac{1}{\phi(t)} \leq \frac{\alpha}{t \phi^{\prime}(t)} \leq \frac{n-1}{t \phi^{\prime}(t)}
$$

and

$$
\begin{aligned}
a & =\mu_{n, \phi}\{|x| \geq r\}=C_{n, \phi} \int_{r}^{+\infty} t^{n-1} e^{-\phi(t)} d t \\
& =C_{n, \phi} \frac{r^{n-1}}{\phi^{\prime}(r)} e^{-\phi(r)}+C_{n, \phi} \int_{r}^{+\infty}\left[\frac{n-1}{t \phi^{\prime}(t)}-\frac{\phi^{\prime \prime}(t)}{\left(\phi^{\prime}(t)\right)^{2}}\right] t^{n-1} e^{-\phi(t)} d t \\
& \geq C_{n, \phi} \frac{r^{n-1}}{\phi^{\prime}(r)} e^{-\phi(r)} \geq C_{n, \phi} r^{n-1} e^{-\phi(r)} \frac{r}{\alpha \phi(r)}
\end{aligned}
$$

Therefore,

$$
\mu^{+}(\partial\{|x| \geq r\})=C_{n, \phi} r^{n-1} e^{-\phi(r)} \leq \alpha a \frac{\phi(r)}{r} .
$$

It remains to bound from above $r$. This is done in two steps. 
The first one consists in proving that $r \geq \phi^{-1}(2 n)$ so that we can use the estimate of Lemma 11. To see that, we complete our lower bound for $a$ by bounding the constant $C_{n, \phi}$ from above:

$$
\begin{aligned}
C_{n, \phi} & =\int_{0}^{+\infty} t^{n-1} e^{-\phi(t)} d t \\
& \leq \int_{0}^{\phi^{-1}(2 n)} t^{n-1} e^{-\phi(t)} d t+\int_{\phi^{-1}(2 n)}^{+\infty} t^{n-1} e^{-\phi(t)} d t \\
& \leq \frac{\left(\phi^{-1}(2 n)\right)^{n}}{n}+\frac{\left(\phi^{-1}(2 n)\right)^{n}}{n} e^{-2 n} \leq 2 \frac{\left(\phi^{-1}(2 n)\right)^{n}}{n} .
\end{aligned}
$$

The upper bound of the second integral comes from our proof of Lemma 11 . Thus, if $r<\phi^{-1}(2 n)$, it holds by (7.2) and (7.3),

$$
a \geq \mu_{n, \phi}\left\{|x| \geq \phi^{-1}(2 n)\right\} \geq \frac{e^{-2 n}}{4 \alpha}
$$

which contradicts the condition $a \leq e^{-3 n}$ as soon as $n \geq \log 4 \alpha$.

Then, we need to reverse the inequality shown at Lemma 11, which was

$$
a \leq F_{n, \phi}(r)=\left(\frac{e r}{\phi^{-1}(n)}\right)^{n} e^{-\phi(r)} .
$$

This is done by Lemma 21 below. So

$$
\mu^{+}(\partial\{|x| \geq r\}) \leq \alpha a \frac{K \log \frac{1}{a}}{\phi^{-1}\left(K \log \frac{1}{a}\right)} \leq \alpha K^{1-1 / \alpha} \frac{a \log \frac{1}{a}}{\phi^{-1}\left(\log \frac{1}{a}\right)} .
$$

Lemma 21. Let $\phi$ be a function satisfying (H0) and $n \in \mathbb{N}^{*}$. Define the function $F_{n, \phi}$ on $\mathbb{R}^{+}$by

$$
F_{n, \phi}(r)=\left(\frac{e r}{\phi^{-1}(n)}\right)^{n} e^{-\phi(r)}
$$

Then, for every $c>0$, there exists $K>0$ such that, whenever $a \leq e^{-c n}$,

$$
F_{n, \phi}(r) \geq a \quad \Longrightarrow \quad r \leq \phi^{-1}\left(K \log \frac{1}{a}\right) .
$$

Proof. First, note that the function $F_{n, \phi}$ is decreasing on $\left(\phi^{-1}(2 n),+\infty\right)$. Thus, whenever $t \geq \phi^{-1}(2 n)$, the inequality $F_{n, \phi}(r) \geq F_{n, \phi}(t)$ implies $r \leq t$. Now, we saw in the proof of Proposition 12 that if $a \leq e^{-c n}$, there exists $K$ large enough such that $t=\phi^{-1}\left(K \log \frac{1}{a}\right)$ satisfies

$$
t \geq \phi^{-1}(2 n) \quad \text { and } \quad F_{n, \phi}(t) \leq \frac{a}{2} .
$$

As $a \leq F_{n, \phi}(r)$, it holds $F_{n, \phi}(r) \geq F_{n, \phi}(t)$ and $r \leq t$ by the preceding remark. 
Acknowledgments. I would like to thank Franck Barthe for his support and fruitful discussions, and also Emanuel Milman for helpful suggestions.

\section{References}

[1] Ané, C., Blachère, S., Chafaï, D., Fougères, P., Gentil, I., Malrieu, F., Roberto, C. And Scheffer, G.: Sur les inégalités de Sobolev logarithmiques. Panoramas et Synthèses 10. Société Mathématique de France, Paris, 2000.

[2] Barthe, F.: Levels of concentration between exponential and Gaussian. Ann. Fac. Sci. Toulouse Math. 10 (2001), no. 3, 393-404.

[3] BARThe, F.: Log-concave and spherical models in isoperimetry. Geom. Funct. Anal. 12 (2002), no. 1, 32-55.

[4] BARThe, F.: Infinite dimensional isoperimetric inequalities in product spaces with the supremum distance. J. Theoret. Probab. 17 (2004), no. 2, 293-308.

[5] Barthe, F., Cattiaux, P. And Roberto, C.: Interpolated inequalities between exponential and Gaussian, Orlicz hypercontractivity and isoperimetry. Rev. Mat. Iberoamericana 22 (2006), no. 3, 993-1067.

[6] Barthe, F., Cattiaux, P. and Roberto, C.: Isoperimetry between exponential and Gaussian. Electron. J. Probab. 12 (2007), no. 44, 1212-1237.

[7] Barthe, F. And Roberto, C.: Sobolev inequalities for probability measures on the real line. Studia Math. 159 (2003), no. 3, 481-497.

[8] Boвkov, S. G.: Extremal properties of half-spaces for log-concave distributions. Ann. Probab. 24 (1996), no. 1, 35-48.

[9] Bobkov, S. G.: Isoperimetric and analytic inequalities for log-concave probability measures. Ann. Probab. 27 (1999), no. 4, 1903-1921.

[10] Bobkov, S. G.: Spectral gap and concentration for some spherically symmetric probability measures. In Geometric aspects of functional analysis, 37-43. Lecture Notes in Math. 1807. Springer, Berlin, 2003.

[11] Borell, C.: Convex measures on locally convex spaces. Ark. Mat. 12 (1974), 239-252.

[12] Gromov, M. and Milman, V. D.: A topological application of the isoperimetric inequality. Amer. J. Math. 105 (1983), no. 4, 843-854.

[13] Huet, N.: Inégalités géométriques pour des mesures log-concaves. $\mathrm{PhD}$ thesis. Université de Toulouse, 2009.

[14] Kannan, R., Lovász, L. And Simonovits, M.: Isoperimetric problems for convex bodies and a localization lemma. Discrete Comput. Geom. 13 (1995), no. 3-4, 541-559.

[15] Klartag, B.: A central limit theorem for convex sets. Invent. Math. 168 (2007), no. 1, 91-131. 
[16] Latala, R. And Oleszkiewicz, K.: Between Sobolev and Poincaré. In Geometric aspects of functional analysis, 147-168. Lecture Notes in Math. 1745. Springer, Berlin, 2000.

[17] Ledoux, M.: The concentration of measure phenomenon. Mathematical Surveys and Monographs 89. American Mathematical Society, Providence, RI, 2001.

[18] Ledoux, M.: Spectral gap, logarithmic Sobolev constant, and geometric bounds. In Surveys in differential geometry. Vol. IX, 219-240. Surv. Differ. Geom., IX. Int. Press, Somerville, MA, 2004.

[19] Milman, E.: On the role of convexity in functional and isoperimetric inequalities. Proc. Lond. Math. Soc. (3) 99 (2009), no. 1, 32-66.

[20] Milman, E.: On the role of convexity in isoperimetry, spectral gap and concentration. Invent. Math. 177 (2009), no. 1, 1-43.

[21] Sodin, S.: An isoperimetric inequality on the $\ell_{p}$ balls. Ann. Inst. $H$. Poincaré Probab. Stat. 44 (2008), no. 2, 362-373.

Recibido: 6 de marzo de 2009

Revisado: 15 de enero de 2010

Nolwen Huet

Institut de Mathématiques de Toulouse UMR CNRS 5219, Université de Toulouse 31062 Toulouse, France nolwen.huet@math. univ-toulouse.fr 